\title{
Magnetic Resonance Imaging Based on Wavelet Algorithm in the Diagnosis and Treatment of Tibial Osteomyelitis Wound Infection
}

\author{
Xiaoran Ou $\mathbb{D}^{1},{ }^{1}$ Qi Wang $\left(\mathbb{D},{ }^{2}\right.$ Chunxiao Li $\mathbb{D},{ }^{1}$ Hongjin Zhao $\mathbb{D},{ }^{1}$ and Lei Guo ${ }^{2}{ }^{2}$ \\ ${ }^{1}$ Department of Orthopedic, No. 920 Hospital of PLA, Kunming 650032, Yunnan, China \\ ${ }^{2}$ Department of Traumatic Orthopedic, Kunming Municipal Hospital of Traditional Chinese Medicine, Kunming 650599, \\ Yunnan, China \\ Correspondence should be addressed to Lei Guo; 201771371@yangtzeu.edu.cn
}

Received 19 September 2021; Revised 3 November 2021; Accepted 6 November 2021; Published 26 November 2021

Academic Editor: M Pallikonda Rajasekaran

Copyright ( 2021 Xiaoran Ou et al. This is an open access article distributed under the Creative Commons Attribution License, which permits unrestricted use, distribution, and reproduction in any medium, provided the original work is properly cited.

\begin{abstract}
This study was to explore the therapeutic effect of magnetic resonance imaging (MRI) images based on the image processing algorithm under the correlation of dyadic wavelet coefficients on the diagnosis of tibial osteomyelitis patients. 32 tibial osteomyelitis patients admitted to hospital were randomly selected as the research objects. According to the patients' wishes, patients who were willing to use new MRI imaging techniques for disease detection were set as the experimental group and conventional MRI imaging detection methods were set as the control group. The application effect of the new MRI imaging technology was evaluated by comparing the treatment effect of the two groups of patients. It was found that the mean square error (MSE) (38.5642) and signal-to-noise ratio (SNR) (18.5122) processed by the improved wavelet algorithm were much better than those of unimproved dyadic wavelet algorithm $(59.1096$ and 15.2341) $(P<0.05)$. The possibilities of soft tissue swelling, bone invasion or destruction, thickening and sclerosis of bone cortex, bone abscess, periosteum response, dense dead bone, and bone sinus of patients in the experimental group were higher than those of the control group, which were $100 \%$ vs. $55 \%, 100 \%$ vs. $80 \%, 92 \%$ vs. $65 \%, 50 \%$ vs. $25 \%, 42 \%$ vs. $15 \%, 67 \%$ vs. $45 \%$, and $50 \%$ vs. $15 \%$, respectively $(P<0.05)$. The healing time of osteomyelitis $(22.89 \pm 2.19 \mathrm{~d}$ vs. $32.32 \pm 2.81 \mathrm{~d})$ and the recovery of wound infection $(14 \%$ vs. $45 \%)$ in the patients in control and experimental groups showed that the results of the experimental group were obviously better than those of the control group. The kappa value of the diagnosis results and tissue biopsy of the experimental group was higher than that of the control group (0.45 vs. 0.34$)$ $(P<0.05)$. In conclusion, the results of the enhanced and improved MRI images were relatively more accurate and the treatment methods adopted were more symptomatic, resulting in more effective treatment. In addition, the wavelet algorithm had certain application value in the enhancement processing of medical images and showed a good development prospect.
\end{abstract}

\section{Introduction}

Osteomyelitis refers to the purulent inflammation involving the bone marrow, bone, and periosteum. It is mainly caused by aerobic or anaerobic bacteria, mycobacteria, and fungi [1]. Osteomyelitis can occur at any age and is most common in children between 2 and 10 years old. The typical clinical manifestations are sudden frequent onsets, chills, high fever, severe pain in the affected limb, refusal to move, and obvious tenderness $[2,3]$. The infection pathways of osteomyelitis mainly include blood-borne dissemination, direct infection after trauma, and direct spread of adjacent soft tissue infections [4]. The main sites of osteomyelitis are the long bones, which are tibia, common bone, humerus, and radius according to the order of incidence. The most common sites in children are long bones with good blood supply, such as the metaphysis of the tibia or femur $[5,6]$. As a very common disease in orthopedic surgery, traumatic tibial osteomyelitis is mainly caused by the infection of bone tissue caused by various traumas, which leads to multiple tissue systems such as periosteum, medullary cavity, and soft tissue [7]. In clinical practice, infection control and complete lesion removal are often used to treat patients with traumatic tibial osteomyelitis. However, after the lesion is removed, bone exposure and surrounding soft tissue defects are likely to occur, which brings certain difficulties to clinical 
treatment [8]. In addition, as a common clinical disease, most cases of osteomyelitis have typical clinical and imaging manifestations. However, the pathogenesis and development of osteomyelitis can be affected by many factors. In particular, the widespread use of antibiotics and changes in bacterial virulence in recent years have led to an increase in osteomyelitis with atypical clinical and imaging findings, making it difficult for imaging diagnosis and differential diagnosis, resulting in misdiagnosis [9]. Magnetic resonance imaging (MRI) has multidirectional imaging capabilities and can provide a lot of information for the diagnosis and treatment of lesions. Noninvasive, painless, and nonradiation ultrasound examination shows high sensitivity and accuracy only for soft tissue lesions, especially suitable for early examination of children with tibial osteomyelitis $[10,11]$.

In recent years, researchers have constantly explored technical methods that can enhance the pixels and resolution of imaging images to make medical imaging technology have more accurate application effects in the examination and diagnosis of clinical diseases. Commonly used image enhancement methods are mainly spatial domain enhancement based on pixel grayscale transformation and frequency domain enhancement based on filtering operations. However, people found that the application of spatial domain enhancement methods for medical image processing often cannot meet the processing requirements of medical images. In the frequency domain enhancement method, because the wavelet transform's multiresolution analysis has good time domain and frequency domain localization characteristics, it can effectively characterize the local characteristics of the signal and focus on the details of the analysis object. Therefore, the wavelet transform is particularly suitable for medical image processing $[12,13]$. A large number of scholars have conducted research and proposed the wavelet exchange algorithm as an image enhancement processing method, but there are not many methods that can be well applicable to the enhancement processing of MRI images [14]. Research has found that the MRI image pixel classification processing algorithm with wavelet coefficient correlation can realize the enhancement of image signals of different scales, better retain the edge characteristics of the image signal, enhance the image detail signal, and suppress the generation of image noise, so it is applicable for processing noise-containing low-contrast MRI images [15].

Therefore, in this study, the image processing algorithm based on correlation of dyadic wavelet coefficients were adopted to enhance the MRI image for the diagnosis of wound infection in patients with tibial osteomyelitis, and the treatment effect of patients with tibial osteomyelitis after the treatment of this technology was observed under the MRI image diagnosis results, aiming to provide more beneficial and improved basis for the clinical diagnosis and treatment of the disease.

\section{Methods}

2.1. Research Objects. In this study, 32 patients with tibial osteomyelitis admitted to hospital from January 2020 to April 2021 were randomly selected as the research objects.
Through soliciting opinions from patients, the patients who were willing to receive the new MRI imaging technology for disease detection were defined as an experimental group, with a total of 12 cases; for the other 20 patients, the conventional MRI imaging methods were adopted for diagnosis and inspection (control group). Among all patients, 19 were males and 13 were females, all aged $21-59$ years. All patients met the clinical diagnostic criteria of tibial osteomyelitis [8]. This study obtained the informed consent of patients and their families, who had signed the informed consent forms in strict accordance with relevant regulations. This study had been approved by the Ethics Committee of hospital.

The inclusion criteria were described as follows: patients with no rejection reaction to MRI examination; patients who could conduct a complete study; and patients with clear mentality and conscious.

The exclusion criteria were defined as follows: pregnant women; patients with other severe liver, kidney, and other organ dysfunctions; and patients with relatively critical illness.

2.2. Conventional MRI Examination Method. All patients undergoing routine MRI examinations used an instrument for examination before and after treatment, which was operated by the same experienced, skilled, and accurate imaging doctor. The superconducting magnetic resonance scanner with a magnetic field strength of $1.5 \mathrm{~T}$ was selected for scanning. The knee joint coil was selected according to the needs of the inspection site (see Figure 1 for the specific operation method), and the image was constructed. Finally, two senior doctors were required to interpret the results. If the results were inconsistent, they had to re-examine and negotiate and finally reach a consensus.

2.3. MRI Imaging Technology Based on the Correlation Processing Algorithm of Dyadic Wavelet Coefficients. MRI imaging technology based on the correlation of dyadic wavelet coefficient processing algorithm was introduced as follows.

In the case of two dimensions, the calculation of the dyadic wavelet transform required multiple wavelet functions in different spatial directions, as shown in equation (1): in general, $d=1$ and $d=2$ represented the horizontal and vertical directions, respectively. The two-dimensional discrete function is shown in equation (2), and its finite-order dyadic wavelet transform can be expressed as equation (3).

$$
\begin{gathered}
\left\{\psi^{q}\right\}_{1 \leq q \leq Q}, \\
f(w, e) \in t^{2}\left(r^{2}\right), \\
Y[f(w, e)]=\left\{Y_{u}^{q}[f(w, e)]_{q=1,2 ; 1 \leq u \leq U}, I_{u}[f(w, e)]\right\} .
\end{gathered}
$$

In the above equations, $\psi$ refers to the dyadic wavelet, $q$ is the discrete value, $\left(r^{2}\right)$ represents the constant value of the function $f(w, e), t^{2}$ is the Fourier transform, and $Y_{u}^{q}[f(w, e)]$ refers to the wavelet transform coefficient of the function $f(w, e)$ at the position $(w, e)$, scale $u$, and direction; 


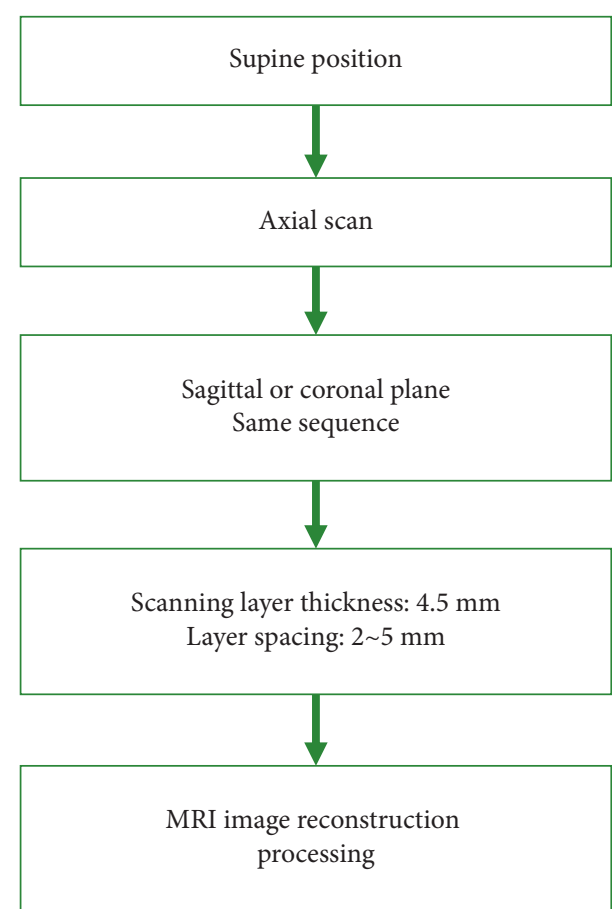

FIgURE 1: The specific operation process of MRI scan.

and $I_{u}[f(w, e)]$ refers to the approximation at the position $(w, e)$ on the maximum order $J$.

Since the signal was dependent on the presence of noise in MRI images, it was required that the selected threshold must have good adaptability. In this study, a new threshold was adopted for calculation, and the expression is given as follows:

$$
\beta=\sqrt{\log \frac{O_{u}}{U} \frac{\alpha_{u}^{2}}{\chi}} .
$$

In equation (4), $U$ is the number of decomposition layers, $O_{u}$ is the width of the subband on the $u$-th scale, $\alpha$ refers to the standard deviation of the noise on the $u$-th order, and $\chi$ represents the variance of the coefficient component matrix on each scale. Because the dyadic wavelet transform was a nonorthogonal transform, the noise energy after the transform cannot be uniformly distributed, so the white noise became colored noise after the transform, and the variance of the noise on each scale had to be estimated. It can be proved by the decomposition structure of wavelet transform [16] that the noise variance of order $u$ is expressed as follows:

$$
\alpha_{u}^{2}=2_{\alpha}^{2}\left|\left(\sum_{p=0}^{u-2} * a_{p}\right) * s^{u-1}\right|_{F} \bullet\left|\left(\sum_{p=0}^{u-2} * a_{p}\right)\right|_{F} .
$$

In the above equation, $\stackrel{2}{\alpha}$ refers to the variance of image noise; $*$ and $\|\bullet\|_{F}$ represent the convolution and norm, respectively; $a_{p}$ and $s^{u-1}$ are filter coefficients. The noise standard deviation of the original MRI image was unknown, so it was necessary to estimate the noise standard deviation, which can be estimated by the wavelet coefficients on the smallest scale, and the estimated value could be calculated with the following equation:

$$
\alpha=\frac{\operatorname{median}\left(\left|Y_{u}^{q} f\right|\right)}{0.6745} .
$$

The confidence method for the improved correlation of wavelet coefficients was introduced as follows. First, the correlation of the wavelet coefficients of adjacent scales is calculated; the wavelet coefficients of the adjacent two scales obtained after multiscale decomposition of the image are $Y_{u}^{q}$ and $Y_{u+1}^{q}$; and the correlation coefficient between the two is given in equation (7). The total power $H_{D_{u}^{q}}$ of the $u$-th correlation coefficient and the total power $H_{Y_{u}^{q}}$ of the wavelet coefficient can be obtained as equations (8) and (9), respectively:

$$
\begin{aligned}
D_{u}^{q} & =Y_{u}^{q} * Y_{u+1}^{q}, \\
H_{D_{u}^{q}} & =\sum_{w, e}\left[D_{u}^{q}(w, e) * D_{u}^{q}(w, e)\right], \\
H_{Y_{u}^{q}} & =\sum_{w, e}\left[Y_{u}^{q}(w, e) * Y_{u}^{q}(w, e)\right] .
\end{aligned}
$$

After normalization, $D_{\mathcal{u}}^{q}$ can be expressed as follows:

$$
\overline{D_{u}^{q}}=D_{u}^{q} * \sqrt{\frac{H_{Y_{u}^{q}}}{H_{D_{u}^{q}}^{q}}} .
$$

Second, the nature of the corresponding points was determined according to the correlation of the wavelet coefficients: in order to determine the cause of the wavelet coefficients, the correlation coefficient plane obtained after normalization was mapped to the confidence plane of the interval $[0,1]$, and the minimum value of $u$-scale correlation coefficient plane could be calculated with the following equation:

$$
\min \overline{D_{u}^{q}}=\min \left\{\left|\overline{D_{u}^{q}(w, e)}\right|\right\} .
$$

Finally, different enhancement methods were selected according to the nature of the point. For the case where the wavelet coefficients were caused by the signal, a linear function was used for enhancement. The expression of the enhancement function is shown as follows:

$$
Y_{\text {out }}= \begin{cases}L * Y_{\text {in }}, & \left|Y_{\text {in }}\right|<K, \\ Y_{\text {in }}+\operatorname{sign}\left(Y_{\text {in }}\right) *(L-1) * K, & \left|Y_{\text {in }}\right| \geq K .\end{cases}
$$

In the above equation, $L$ refers to the wavelet enhancement coefficient and $K$ represents the wavelet coefficient threshold. Since the enhanced part here was clearly an image signal, the linear stretching of the signal's contrast would not cause the amplification of noise.

If the wavelet coefficients were caused by noise, a hardthreshold denoising function was used, as shown in the following equation:

$$
Y_{\text {out }}= \begin{cases}0, & \left|Y_{\text {in }}\right|<K, \\ Y_{\text {in }}, & \left|Y_{\text {in }}\right|>K\end{cases}
$$


The mean square error (MSE) and signal-to-noise ratio (SNR) were adopted to evaluate the enhancement effect after processing, and the expression equations are as follows:

$$
\begin{aligned}
& \text { MSE }=\frac{1}{Q \bullet W} \sum_{q=1, w=1}^{Q, W}\left(f_{q, w}-\overline{f_{q, w}}\right)^{2}, \\
& \mathrm{SNR}=10 \lg \left[\frac{\sum_{q=1, w=1}^{Q, W} f^{2} m, n}{\sum_{q=1, w=1}^{Q, W}\left(f_{q, w}-\overline{f_{q, w}}\right)^{2}}\right] .
\end{aligned}
$$

The smaller the variance MSE and the larger the signalto-noise ratio, the better the processing effect.

2.4. Observation Indicators. The MRI imaging results of the two groups of patients were compared, including the lesions of the soft tissue, bone, periosteum, cortical bone, bone marrow, and bone marrow cavity of the lesion.

The treatment effects of the two groups of patients were compared, and a comprehensive evaluation was made by comparing the functional activities of the knee and ankle joints, the wound healing time, the control of wound infection, and the clinical treatment effect of the two groups of patients after treatment [17]. Both the Hospital for Special Surgery Knee Score (HSS score) [18] and American Orthopaedic Foot and Ankle Society (AOFAS) [19] score were 100 points. The higher the score, the better the patient's knee and ankle joint function. For the wound infection control: if the wound color was ruddy, there was no purulent secretion and no peculiar smell, it was better; otherwise, no improvement was seen.

2.5. Statistical Analysis. The SPSS22.0 statistical software system was applied for data entry, sorting, and statistical analysis. The comparison of count data was performed using the $X^{2}$ test, and the comparison of measurement data was performed using the $t$ test. Multiple sample means were compared by analysis of variance, the Least Significant Difference (LSD) method was used when the variance was uniform, and the Dunnett T3 method was used when the variance was uneven. $P<0.05$ meant that there was a statistically significant difference. The kappa test was performed on the consistency between the MRI image inspection result and the tissue biopsy. When kappa $>0.75$, the consistency between the two was strong; when $0.4 \leq$ kappa $<0.75$, the consistency between the two was general; and when kappa $<0.4$, the consistency between the two was poor.

\section{Results}

3.1. Algorithm Processing Effect. Figure 2 shows the comparison of the processing effect of the improved dyadic wavelet algorithm and the improved dyadic wavelet coefficient correlation processing algorithm on MRI images. According to the results, the image MSE (38.5642) and SNR (18.5122) processed by the improved wavelet algorithm were much better than those of the unimproved dyadic wavelet algorithm $(59.1096$ and 15.2341) $(P<0.05)$. In

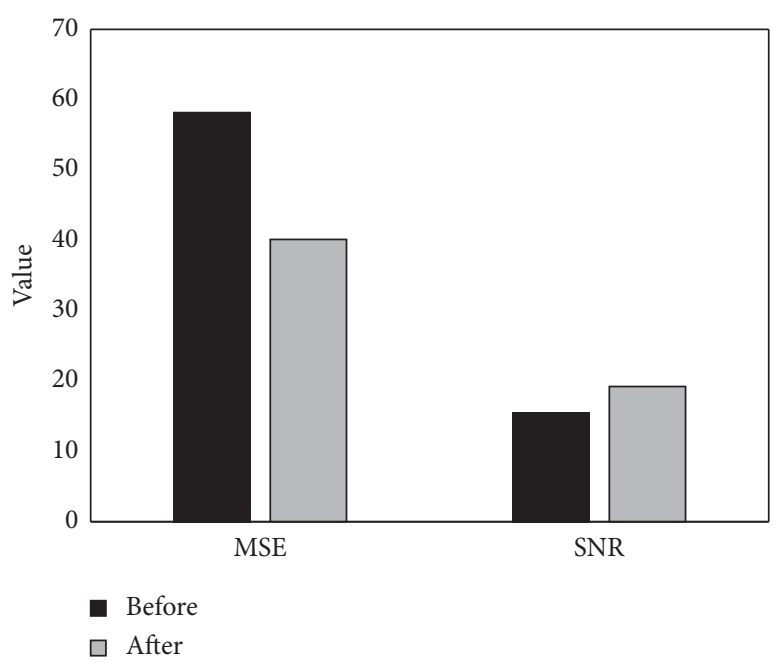

FIgure 2: Comparison on MRI image processing effect of wavelet algorithm before and after improvement.

Figure 3, the comparison of MRI images before and after using the dyadic wavelet coefficient correlation processing algorithm is shown. It illustrated that the resolution of the processed image was greatly improved.

3.2. Comparison on Basic Data of Patients in Two Groups. During this study, the statistics were performed firstly on general data of the two groups of randomly selected patients. Of the 12 patients in the experimental group, the numbers of males and females were both 6 , and the numbers of males and females of the 20 patients in the control group were 11 and 9 , respectively. The proportions of men in the two groups were $50 \%$ and $55 \%$, respectively, and the proportions of women were $50 \%$ and $45 \%$, respectively. Through observation shown in Table 1, it was found that there was no significant difference in the age range and average age of the two groups of patients, and the above comparison was not statistically significant $(P>0.05)$. Therefore, it was feasible to study and compare the two groups of patients.

\subsection{The Results of MRI Imaging for Tibial Osteomyelitis in the} Control Group. After the routine MRI imaging examination, the patients in the control group were analyzed by professional doctors and the results were statistically sorted. Figure 4 shows the distribution results of the soft tissue, bone, periosteum, cortical bone, bone marrow, and bone marrow cavity of the patients with acute tibial osteomyelitis and chronic tibial osteomyelitis in the control group. It indicated that the majority of patients with acute tibial osteomyelitis had the above lesions. Figure 5 shows the MRI images of the same patient's lesions in different orientations, including coronal, sagittal, and axial lesions.

3.4. MRI Images of the Experimental Group to Check the Condition of Lower Tibial Osteomyelitis. The patients in the experimental group were examined through the MRI images processed by the improved dyadic wavelet coefficient 


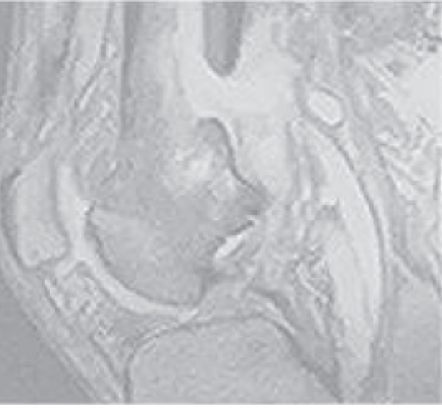

(a)

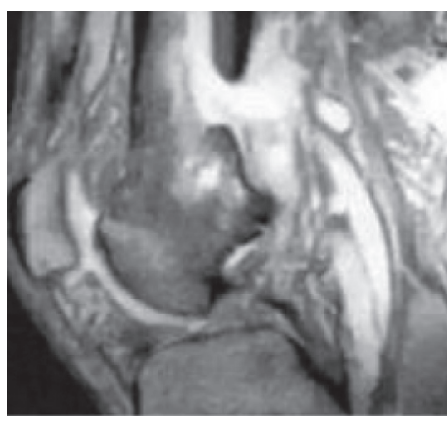

(b)

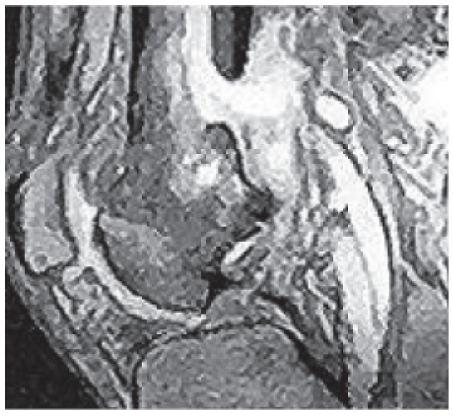

(c)

FIGURE 3: Comparison on MRI images processed by wavelet algorithm. (a) Original image, (b) image processed by wavelet algorithm before improvement, and (c) image processed by wavelet algorithm after improvement.

TABle 1: Comparison on basic data of patients in two groups.

\section{Gender}

Age

Males $(n=19)$ Proportion (\%) Females $(n=13)$ Proportion (\%) Age range (years) Average age (years)

\begin{tabular}{lcccccc}
\hline Experimental group $(n=12)$ & 6 & 50 & 6 & 50 & {$[21,57]$} & $(37.78 \pm 2.36)$ \\
Control group $(n=20)$ & 11 & 55 & 9 & 45 & {$[22,59]$} & $(38.21 \pm 3.09)$ \\
\hline
\end{tabular}

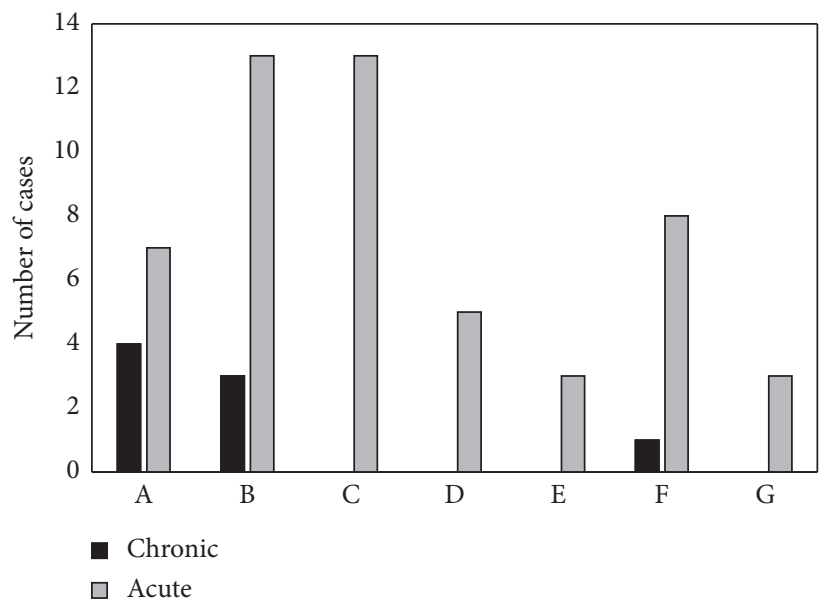

Figure 4: The lesions of tibial osteomyelitis patients in the control group. Note. A-G refer to soft tissue swelling, bone invasion or destruction, cortical thickening and hardening, bone abscess, periosteal reaction, compact sequestrum, and sinus tract, respectively.
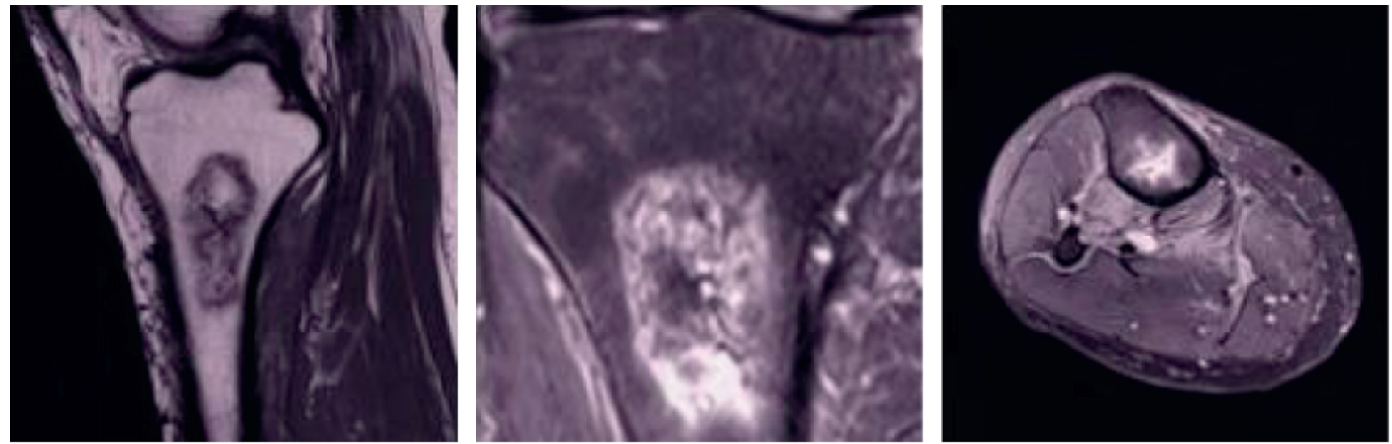

FIGURE 5: MRI images of tibial osteomyelitis in different directions of patients in the control group.

correlation processing algorithm, and the results were statistically sorted after analysis by professional physicians. Figure 6 shows the distribution results of the soft tissue, bone, periosteum, cortical bone, bone marrow, and bone marrow cavity of the patients with acute tibial osteomyelitis and chronic tibial osteomyelitis in the control group. It 


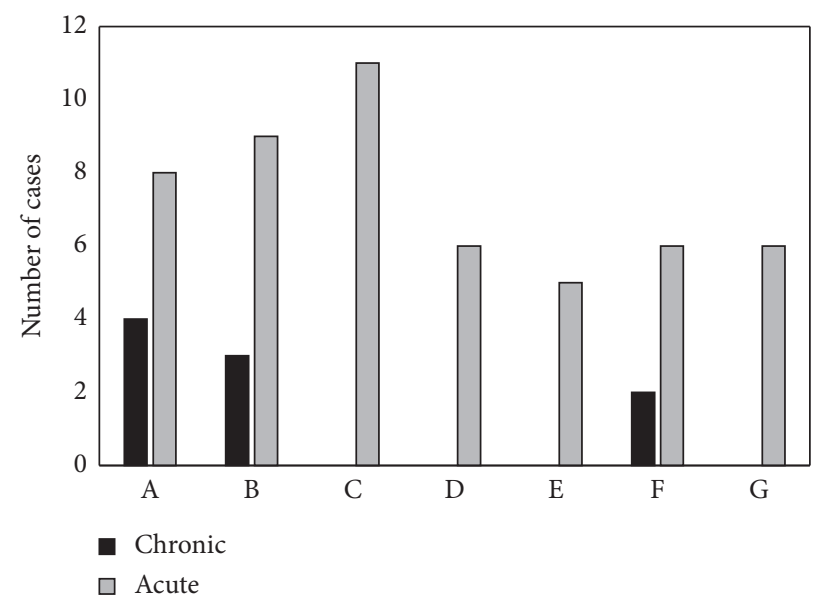

Figure 6: The lesions of tibial osteomyelitis patients in the experimental group. Note. A-G refer to soft tissue swelling, bone invasion or destruction, cortical thickening and hardening, bone abscess, periosteal reaction, compact sequestrum, and sinus tract, respectively.

indicated that the majority of patients with acute tibial osteomyelitis had the above lesions. Figure 7 shows the MRI images of the same patient's lesions in different orientations in the experimental group, including coronal, sagittal, and axial lesions.

3.5. Comparison of the Results of the Two Groups of MRI Images. As illustrated in Figure 8, the comparison on the above two MRI examination results and images revealed that the possibilities of all patients in the experimental group detected soft tissue swelling, bone invasion or destruction, cortical thickening and hardening, bone abscess, periosteal reaction, compact sequestrum, and sinus tract were higher compared to those of the control group, showing statistically significant differences $(P<0.05)$. Comparison on MRI images of the two groups of patients (Figure 9) suggested that the MRI image in the experimental group that was enhanced by the correlation of dyadic wavelet coefficients processing algorithm was obviously higher than that in the control group in terms of pixels and resolution, and the high and low signal boundaries of the lesion were more obvious. The boundary between the lesion of patient in the control group and normal tissue was relatively blurred. The above results indicated that the inspection results of the enhanced and improved MRI image were relatively more accurate.

3.6. Comparison on Treatment Status and the Consistency between the Results of the Needle Biopsy and the MRI Examination of the Two Groups of Patients. The healing time and wound infection recovery of the two groups of patients were observed and compared, and the results are given in Table 2. In terms of the patient's leg activity and treatment time, the evaluation result of experimental group was greatly higher and the treatment time was obviously less in contrast to the control group, and the comparison was statistically significant $(P<0.05)$. The wound infection probability and recurrence probability of the experimental group were dramatically lower than those of the control group, and the comparison was statistically significant $(P<0.05)$. After that, the kappa test was performed based on the consistency between the MRI diagnosis result and the tissue biopsy result. The result suggested that the kappa value of the experimental group diagnosis result and the tissue biopsy was 0.45 , while the kappa value of the control group was 0.34 , so in the experimental group, it was much higher, showing statistically observable difference $(P<0.05)$. Therefore, it showed that the diagnostic results of experimental group patients were relatively more accurate and the treatment methods adopted were more symptomatic, so that more effective treatment can be achieved.

\section{Discussion}

In recent years, with the abuse of antibiotics and the continuous changes in bacterial toxicity and the enhancement of drug resistance, the number of patients with clinically atypical clinical manifestations and imaging changes in osteomyelitis has also increased, leading to the difficulty in imaging diagnosis, and differential diagnosis is increased, which often causes misdiagnosis and delays the timely treatment of the disease [20]. Therefore, in order to improve the accuracy of diagnosis, experts are constantly researching and exploring. At this stage, the commonly used imaging examinations for the diagnosis of osteomyelitis include plain X-ray, CT, MRI, ultrasound, and radionuclide bone scan. $\mathrm{X}$-ray manifestations in the early stage of the disease are mostly negative, which is of little significance for the early diagnosis of the disease. The radionuclide bone scan can show abnormal radioactive concentration in the early stage of the disease, with high sensitivity, but poor specificity and high false negatives. CT and MRI have good soft tissue resolution, which can detect lesions early and accurately display the extent of lesions [21-24]. To understand the value of MRI in the diagnosis of osteomyelitis, a retrospective analysis of the X-ray, MRI plain scan, and Gd-DTPA enhanced scan data of 20 patients with osteomyelitis showed that MRI had important value in the diagnosis of osteomyelitis, especially in showing early lesions and the scope of the lesion was significantly better than X-ray [25]. Some 


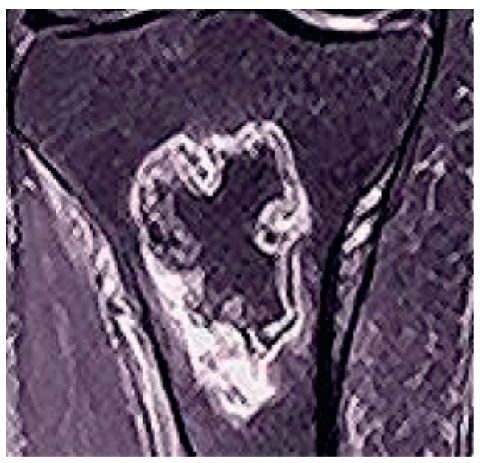

Coronal view

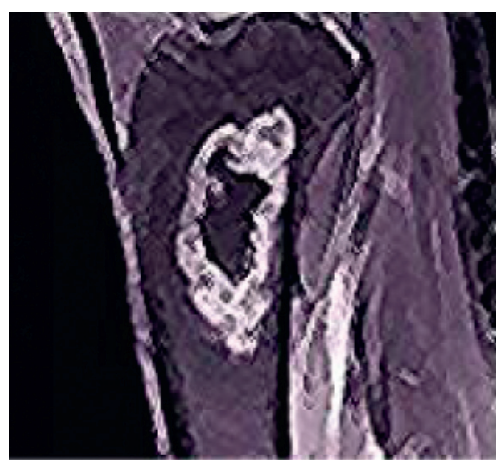

Sagittal

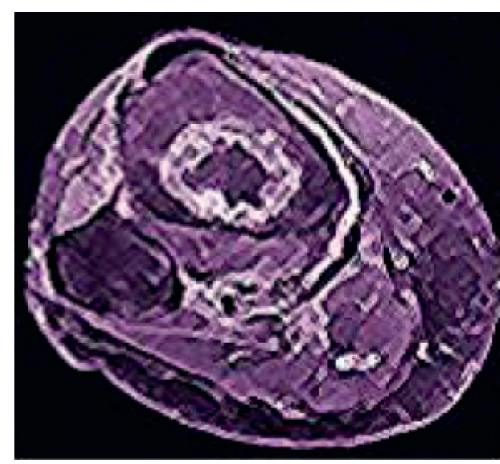

Axial position

FIgURE 7: MRI images of tibial osteomyelitis in different directions of patients in the experimental group.

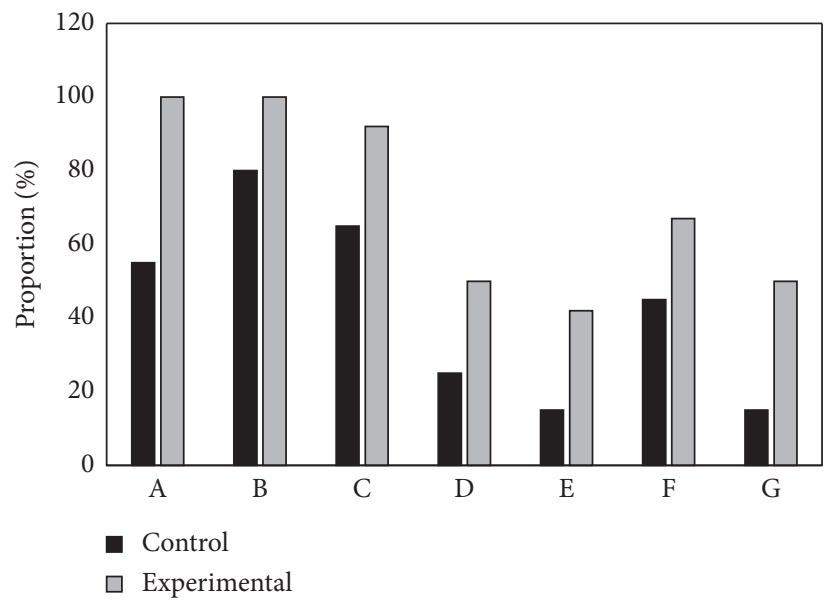

Figure 8: Comparison on the proportions of symptoms detected in the lesions of the two groups of patients with tibial osteomyelitis. Note. A-G represent soft tissue swelling, bone invasion or destruction, cortical thickening and hardening, bone abscess, periosteal reaction, compact sequestrum, and sinus tract, respectively.

people also compared the X-ray, CT, and MRI manifestations of osteomyelitis, focusing on the MRI manifestations of various signs of osteomyelitis and the application value of various imaging examination methods to osteomyelitis; it was found that MRI can accurately estimate the extent of the lesion, which was larger than the X-ray examination; in addition, its high soft tissue resolution was incomparable with X-ray and CT, which could clearly display intramedullary and extramedullary lesions, basically meeting the clinical requirements for modern imaging medicine; it was concluded that, for the early diagnosis and treatment of osteomyelitis, MRI was the most valuable imaging method [26]. In this study, the improved method of wavelet algorithm (dyadic wavelet coefficient correlation processing algorithm) was used to enhance the MRI image of the experimental group of patients and used for disease diagnosis and treatment. A series of examinations and the healing of the patients in the later period were observed and compared. First, it was concluded that the image MSE and SNR processed by the improved wavelet algorithm were much better than those of the unimproved dyadic wavelet algorithm $(P<0.05)$. Some research experts have proposed a new noise suppression algorithm based on dyadic wavelet transform for the shortcomings of traditional denoising methods that blur the edges of MRI images while reducing noise, and experimental results revealed that this method showed higher denoising accuracy than traditional denoising methods and could effectively reduce noise while maintaining image details and edge information [16]. A third-order piecewise function-based unfixed point processing algorithm is also proposed. Experiments show that this method can achieve pixel classification, suppress noise points, and effectively enhance target pixels [27]. Wu et al. [28] also proposed in their research that compared with the traditional wavelet transform, the improved wavelet transform algorithm can capture more geometric features of the spherical image. The above studies all support the results of this study. In this study, the consistency of the results of the needle biopsy and MRI examinations of the two groups of patients was compared, and the kappa value of the diagnosis results and tissue biopsy of the experimental group was higher than that of the control group $(P<0.05)$. In the healing time of osteomyelitis and the recovery of wound infection in the two groups of patients, the results of the experimental group were obviously better than those of the control group. It shows that as the display resolution ratio of 


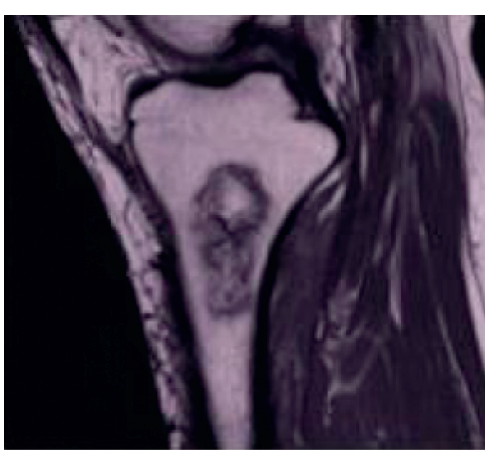

Coronal view

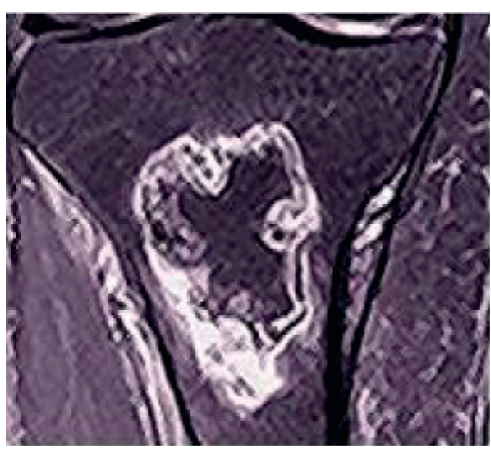

Coronal view

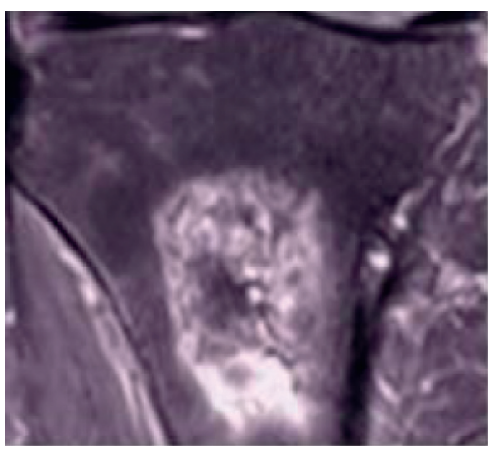

Sagittal

(a)

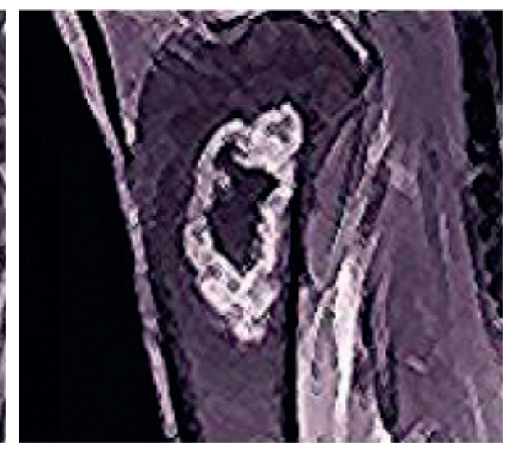

Sagittal

(b)

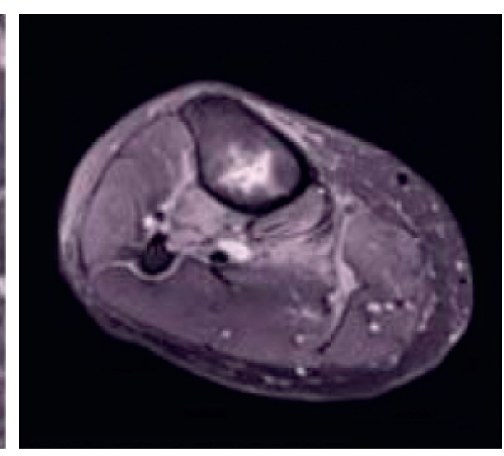

Axial position

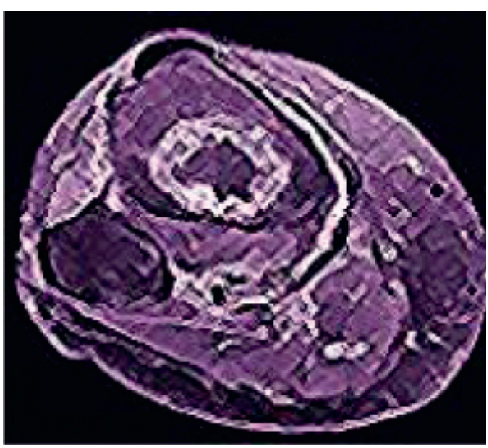

Axial position

FIGURE 9: MRI images of two groups of patients.

TABle 2: Comparison of the treatment status of the two groups of patients.

\begin{tabular}{lcc}
\hline & Experimental group & Control group \\
\hline HSS score & $80.45 \pm 5.13$ & $59.67 \pm 3.18$ \\
AOFAS score & $78.01 \pm 3.12$ & $43.23 \pm 4.98$ \\
Wound healing time (d) & $22.89 \pm 2.19$ & $32.32 \pm 2.81$ \\
Hospital stays (d) & $34.78 \pm 2.91$ & $28.89 \pm 2.10$ \\
Wound infection rate (\%) & 89 & 61 \\
Incidence (\%) & 14 & 45 \\
\hline
\end{tabular}

the MRI image is higher, the diagnosis result is closer to the accurate result and the treatment effect for the patient is better. Such results are consistent with the research results of Lai et al. [29].

\section{Conclusion}

In this study, the MRI images processed by the correlation of dyadic wavelet coefficient algorithm were applied to diagnose the wound infection in patients with tibial osteomyelitis, aiming to observe the treatment effect. The results of the study are summarized as follows. All patients in the experimental group showed higher probabilities of detecting soft tissue swelling, bone invasion or destruction, cortical thickening and hardening, bone abscess, periosteal reaction, compact sequestrum, and sinus tract in contrast to those of the control group; in the healing time of osteomyelitis and wound infection recovery of the two groups of patients, the results of experimental group were obviously better than those of control group, and the kappa values of experimental group diagnosis results and tissue biopsy were higher than those of control group.

Based on the above results, it was found that the examination results of the enhanced and improved MRI images were relatively more accurate and the treatment methods adopted were more symptomatic, thus achieving more effective treatment. It suggested that the wavelet algorithm had a reliable application value in the enhancement processing of medical images and showed a good development prospect.

\section{Data Availability}

The data used to support the findings of this study are available from the corresponding author upon request.

\section{Conflicts of Interest}

The authors declare that there are no conflicts of interest.

\section{References}

[1] S. K. Schmitt, "Osteomyelitis," Infectious Disease Clinics of North America, vol. 31, no. 2, pp. 325-338, 2017, PMID: 28483044.

[2] L. Castellazzi, M. Mantero, and S. Esposito, "Update on the management of pediatric acute osteomyelitis and septic 
arthritis," International Journal of Molecular Sciences, vol. 17, no. 6, p. 855, 2016 PMID: 27258258; PMCID: PMC4926389.

[3] N. Maffulli, R. Papalia, B. Zampogna, G. Torre, E. Albo, and V. Denaro, "The management of osteomyelitis in the adult," The Surgeon, vol. 14, no. 6, pp. 345-360, 2016, Epub 2016 Jan 21. PMID: 26805473.

[4] J. C. Mandell, B. Khurana, J. T. Smith, G. J. Czuczman, V. Ghazikhanian, and S. E. Smith, "Osteomyelitis of the lower extremity: pathophysiology, imaging, and classification, with an emphasis on diabetic foot infection," Emergency Radiology, vol. 25, no. 2, pp. 175-188, 2018, Epub 2017 Oct 20. PMID: 29058098.

[5] Z. Q. Wu, D. L. Zeng, J. L. Yao et al., "Research progress on diagnosis and treatment of chronic osteomyelitis," Chinese Medical Sciences Journal, vol. 34, no. 3, pp. 211-220, 2019, PMID: 31601304.

[6] Z. Chen, L. Cheng, and G. Feng, "Bone inflammation and chronic recurrent multifocal osteomyelitis," European Review for Medical and Pharmacological Sciences, vol. 22, no. 5, pp. 1380-1386, 2018, PMID: 29565497.

[7] L. Xiao, T. Li, M. Ding et al., "Detecting chronic post-traumatic osteomyelitis of mouse tibia via an IL-13r $\alpha 2$ targeted metallofullerene magnetic resonance imaging probe," Bioconjugate Chemistry, vol. 28, no. 2, pp. 649-658, 2017, Epub 2017 Feb 6. PMID: 28061526; PMCID: PMC5317096.

[8] X. Yikemu, A. Tuxun, M. Nuermaimaiti, A. Abudukeyimu, and A. Shayiti, "Effects of vacuum sealing drainage combined with ilizarov bone transport technique in the treatment of tibial traumatic osteomyelitis," Medical Science Monitor, vol. 25, pp. 6864-6871, 2019, PMID: 31513555; PMCID: PMC6754706.

[9] J. Fattore, D. S. L. Goh, A. Al-Hindawi, and D. Andresen, "Revisiting the important role of magnetic resonance imaging (MRI) in long bone acute osteomyelitis: a case report of methicillin resistant Staphylococcus aureus acute tibial osteomyelitis with conventional radiography, computed tomography, and MRI," Radiol Case Rep, vol. 15, no. 10, pp. 2003-2008, 2020, Epub 2020 Aug 22. PMID: 32864031; PMCID: PMC7443062.

[10] S. Andronikou, T. Mendes da Costa, M. Hussien, and A. V. Ramanan, "Radiological diagnosis of chronic recurrent multifocal osteomyelitis using whole-body MRI-based lesion distribution patterns," Clinical Radiology, vol. 74, no. 9, pp. 737.e3-737.e15, 2019, Epub 2019 Jul 4. PMID: 31280883.

[11] Y. Zhao, T. S. Sato, S. M. Nielsen et al., "Development of a scoring tool for chronic nonbacterial osteomyelitis magnetic resonance imaging and evaluation of its interrater reliability," Journal of Rheumatology, vol. 47, no. 5, pp. 739-747, 2020, Epub 2019 Oct 1. PMID: 31575701.

[12] E. Shimron, A. G. Webb, and H. Azhari, "CORE-PI: noniterative convolution-based reconstruction for parallel MRI in the wavelet domain," Medical Physics, vol. 46, no. 1, pp. 199-214, 2019, Epub 2018 Nov 20. PMID: 30365167.

[13] M. N. Ali, "A wavelet-based method for MRI liver image denoising," Biomedizinische Technik, vol. 64, no. 6, pp. 699709, 2019, PMID: 31145685.

[14] J. Bhardwaj and A. Nayak, "Haar wavelet transform-based optimal Bayesian method for medical image fusion," Medical, \& Biological Engineering \& Computing, vol. 58, no. 10, pp. 2397-2411, 2020, Epub 2020 Jul 30. PMID: 32734326.

[15] R. R. Ayalapogu, S. Pabboju, and R. R. Ramisetty, "Analysis of dual tree M-band wavelet transform based features for brain image classification," Magnetic Resonance in Medicine, vol. 80, no. 6, pp. 2393-2401, 2018, Epub 2018 Apr 29. PMID: 29707806.

[16] M. Zhou, D. Badre, and H. Kang, "Double-wavelet transform for multisubject task-induced functional magnetic resonance imaging data," Biometrics, vol. 75, no. 3, pp. 1029-1040, 2019, Epub 2019 Apr 17. PMID: 30985916; PMCID: PMC6771256.

[17] J. Peng, Y. Ren, W. He et al., "Epidemiological, clinical and microbiological characteristics of patients with post-traumatic osteomyelitis of limb fractures in southwest China: a hospital-based study," J Bone Jt Infect, vol. 2, no. 3, pp. 149-153, 2017, PMID: 28540152; PMCID: PMC5441147.

[18] H. Sun, L. Zhou, F. Li, and J. Duan, "Comparison between closing-wedge and opening-wedge high tibial osteotomy in patients with medial knee osteoarthritis: a systematic review and meta-analysis," Journal of Knee Surgery, vol. 30, no. 2, pp. 158-165, 2017, Epub 2016 May 24. Erratum in: J Knee Surg. 2017 Feb;30(2):e1. PMID: 27218480.

[19] B. Vahdatpour, H. Forouzan, F. Momeni, M. Ahmadi, and P. Taheri, "Effectiveness of extracorporeal shockwave therapy for chronic Achilles tendinopathy: a randomized clinical trial," Journal of Research in Medical Sciences, vol. 23, p. 37, 2018 PMID: 29887905; PMCID: PMC5961283.

[20] E. García Del Pozo, J. Collazos, J. A. Cartón, D. Camporro, and V. Asensi, "Bacterial osteomyelitis: microbiological, clinical, therapeutic, and evolutive characteristics of $344 \mathrm{ep}-$ isodes," Revista Española de Quimioterapia, vol. 31, no. 3, pp. 217-225, 2018, Epub 2018 May 11. PMID: 29756429; PMCID: PMC6166254.

[21] I. J. E. Kouijzer, H. Scheper, J. W. J. de Rooy et al., "The diagnostic value of 18F-FDG-PET/CT and MRI in suspected vertebral osteomyelitis-a prospective study," European Journal of Nuclear Medicine and Molecular Imaging, vol. 45, no. 5, pp. 798-805, 2018, Epub 2017 Dec 19. PMID: 29256136; PMCID: PMC5978906.

[22] A. G. Jurik, R. F. Klicman, P. Simoni, P. Robinson, and J. Teh, "SAPHO and CRMO: the value of imaging," Seminars in Musculoskeletal Radiology, vol. 22, no. 2, pp. 207-224, 2018, Epub 2018 Apr 19. PMID: 29672809.

[23] Z. Xia, C. Wang, A. Arnold et al., "Efficacy of treating chronic tibial osteomyelitis with bone defect using a pedicled perforator-layered flap and fasciocutaneous flap of the posterior tibial artery: a case report," Wounds, vol. 32, no. 11, pp. E50-E54, 2020, PMID: 33465040.

[24] K. Tran and M. Mierzwinski-Urban, Serial X-Ray Radiography for the Diagnosis of Osteomyelitis: A Review of Diagnostic Accuracy, Clinical Utility, Cost-Effectiveness, and Guidelines [Internet], Canadian Agency for Drugs and Technologies in Health, Ottawa (ON), PMID: 33074603, 2020.

[25] K. Gariani, D. Lebowitz, B. Kressmann, J. Gariani, and I. Uçkay, "X-Ray versus magnetic resonance imaging in diabetic foot osteomyelitis: a clinical comparison," Current Diabetes Reviews, vol. 17, no. 3, pp. 373-377, 2021, PMID: 32729421.

[26] C. L. Ramanujam, D. Han, and T. Zgonis, "Medical imaging and laboratory analysis of diagnostic accuracy in 107 consecutive hospitalized patients with diabetic foot osteomyelitis and partial foot amputations," Foot \& Ankle Specialist, vol. 11, no. 5, pp. 433-443, 2018, Epub 2017 Dec 31. PMID: 29291264.

[27] G. Baldazzi, G. Solinas, J. Del Valle et al., "Systematic analysis of wavelet denoising methods for neural signal processing," Journal of Neural Engineering, 2020, Epub ahead of print. PMID: 33142283. 
[28] G. Wu, Y. Shi, X. Sun, J. Wang, and B. Yin, "SMSIR: spherical measure based spherical image representation," IEEE Transactions on Image Processing, vol. 30, pp. 6377-6391, 2021, Epub 2021 Jul 14. PMID: 34003750.

[29] Z. Lai, X. Zhang, D. Guo et al., "Joint sparse reconstruction of multi-contrast MRI images with graph based redundant wavelet transform," BMC Medical Imaging, vol. 18, no. 1, p. 7, 2018 PMID: 29724180; PMCID: PMC5934877. 